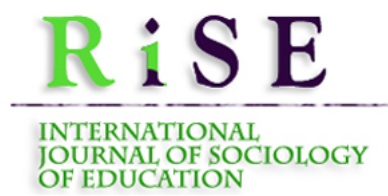

Instructions for authors, subscriptions and further details:

http://rise.hipatiapress.com

\title{
Liderazgo en la Escuela Rural: Estudios de Casos
}

Dolors Mayoral Arqué ${ }^{1}$

Joana Colom ${ }^{2}$

Olga Bernad ${ }^{1}$

Teresa Torres González ${ }^{1}$

1) Universitat de Lleida, Spain

2) Universidad de las Islas Baleares, Spain

Date of publication: February $25^{\text {th }}, 2018$

Edition period: February 2018-June 2018

To cite this article: Mayoral Arqué, D., Colom, J., Bernad, O. \& Torres González, T. (2018). Liderazgo en la Escuela Rural: Estudios de Casos, International Journal of Sociology of Education, 7(1), 49-70. doi:

10.17583/rise.2018.2637

To link this article: http://dx.doi.org/10.17583/rise.2018.2637

PLEASE SCROLL DOWN FOR ARTICLE

The terms and conditions of use are related to the Open Journal System and to Creative Commons Attribution License (CC-BY) 


\title{
Leadership in the Rural School: Case Studies
}

Dolors Mayoral Arqué

Universitat de Lleida

Olga Bernad

Universitat de Lleida
Joana Colom

Universidad de las Islas Baleares

Teresa Torres González

Universitat de Lleida

(Received: 13 March 2017; Accepted: 16 November 2017; Published: 25 February 2018)

\begin{abstract}
We studied the construction of leadership in teachers who work as headmasters and head mistresses at rural schools in Catalonia. The objective was to analyse the emergence and development of leadership. We also sought to investigate the possible existence of differentiated models of leadership amongst rural teachers. This study was based on the case analysis approach. To carry it out, we chose four cases, involving two headmasters and two headmistresses, who stood out as the leaders of unique and highly innovative projects.. It was found that leadership was the result of a complex process that required a favourable environment, a high level of motivation, skills and a vision of the future that made it possible to construct differentiated projects and to reach goals and objectives associated with innovative criteria within the field of education. These schools could be defined as places where people learn; this means that the students learn, the teachers learn, the families learn and the community learns. In contrast to the traditional conception of the charismatic leader, the leader is not born, but is made. Furthermore, leadership is shared, distributed and collaborative. However, the most relevant thing about these leaders is their social commitment and their values; they not only seek to contribute to educational change but also try to help improve the rural communities in which they carry out their projects. It is therefore necessary to speak not only of ethical leadership but also of social leadership.
\end{abstract}

Keywords: rural education, rural schools, education and school, primary schools, teacher leadership, teaching innovation

2018 Hipatia Press

ISSN: 2014-3575

DOI: $10.17583 /$ rise.2018.2637 


\section{Liderazgo en la Escuela Rural: Estudios de Casos}

Dolors Mayoral Arqué

Universitat de Lleida

Olga Bernad

Universitat de Lleida
Joana Colom

Universidad de las Islas Baleares

Teresa Torres González

Universitat de Lleida

(Recibido: 13 Marzo 2017; Aceptado: 16 Noviembre 2017; Publicado: 25 Febrero 2018)

\section{Resumen}

Este artículo estudia la construcción del liderazgo en maestros y maestras que desarrollan su tarea como directivos y directivas en escuelas rurales de Cataluña. El objetivo se centró en analizar la emergencia y desarrollo del liderazgo. Asimismo, se indagó sobre la posible existencia de modelos de liderazgo diferenciados en maestros y maestras rurales. El estudio se basa en la metodología de análisis de casos. Para ello escogimos cuatro casos, específicamente dos maestros y dos maestras directivos que destacaban por liderar proyectos singulares e innovadores; cuestión que permitió conocer con mayor profundidad los aspectos de esta investigación. Se constató que el liderazgo es el resultado de un proceso complejo que requiere de un entorno favorable, una elevada motivación, habilidades y una visión de futuro que permita construir proyectos diferenciados y alcanzar metas y objetivos, respondiendo a criterios de innovación en el ámbito de la educación. Estos centros se definen como escuelas que aprenden, es decir, aprende el alumnado, aprende el profesorado, aprenden las familias $y$ aprende la comunidad. Contrariamente a la concepción tradicional del líder carismático, el líder no nace, se hace. Además, el liderazgo se comparte, se distribuye y es colaborativo. Pero lo más importante de estos y estas líderes es su compromiso social y sus valores, ya que no sólo pretenden contribuir al cambio educativo sino también aportar una mejora a la comunidad rural en la que desarrollan su proyecto, por lo que cabe hablar no solamente de liderazgo ético sino también de liderazgo social.

Palabras clave: escuelas rurales, educación y escuela, educación primaria, maestros líderes, innovación docente

2018 Hipatia Press

ISSN: 2014-3575

DOI: $10.17583 /$ rise.2018.2637

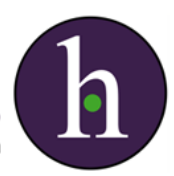


a temática del liderazgo es relevante y está presente en los contextos educativos. El liderazgo escolar se ha convertido en una prioridad de los programas de política educativa a nivel internacional por ser decisivo en la mejora de los resultados escolares e influir en las motivaciones, en las capacidades del profesorado y en el ambiente escolar (OCDE, 2009). Igualmente, La Red de Liderazgo Escolar de la OREALC/UNESCO resalta la importancia de los directivos y directivas en la mejora del aprendizaje.

De acuerdo con Rojas \& Gaspar (2006) la palabra "líder" significa "guiar" o "conducir". Asensio Aguilera (2013) enfatiza las dimensiones democráticas, inclusivas y de equidad social del ejercicio del liderazgo. Para Losada (2007), ejercer liderazgo significa principalmente orientar y motivar. Una buena práctica de liderazgo en el centro crea equipo, favorece unos valores y un saber hacer compartidos, y ayuda al desarrollo de una cultura docente organizativo-colaborativa.

Conviene recordar que liderar no es equivalente a dirigir (Frederickson \& Smith, 2003; Fry, 1989; Guillem, 1994; Mintzberg, 1983). Dirigir conlleva utilizar recursos para lograr objetivos; significa también evaluar, gestionar y controlar, actividades todas relacionadas con la gestión.

Un líder es el que establece una interrelación o influencia mutua entre un grupo de personas o equipo colaborador, seguidores en un contexto, mediante una visión que es asumida voluntariamente y que es atractiva para la propia vida. De acuerdo con Castiñeira \& Lozano (2012), estos autores definen dos modelos de liderazgo: el gerencial y el liderazgo ético; cualquiera de estos dos modelos puede aparecer en organizaciones e instituciones educativas.

En este artículo se va analizar la emergencia del liderazgo en los maestros y maestras de escuela rural, cómo se desarrolla y si existen unas características diferenciales entre los maestros y maestras líderes.

\section{Liderazgo Educativo}

Bolívar (2012) entiende el liderazgo en el ámbito educativo como la capacidad de influir en las personas, con el fin de que éstas adopten líneas para desarrollar una acción. A diferencia del modelo de dirección tradicional basado en el poder de la dirección, Bolívar propone una dirección 


\section{Mayoral et al. - Liderazgo en la Escuela Rural}

compartida; es decir, donde se delegan las acciones. Este autor enfatiza la importancia de cambiar aquel modelo de liderazgo centrado en la burocracia por un modelo más innovador, abierto, flexible y compartido.

Por otra parte, Gallego, González \& Brugué (2011) indican que "el liderazgo educativo parte de la reflexión sobre el rol del director como líder, sus funciones, capacidades, objetivos y responsabilidades en el marco de una institución (la escuela) que se encuentra en constante cambio y necesitada de nuevos instrumentos de mejora". De la tesis de estos autores se desprende que el liderazgo educativo se encuentra vinculado a la innovación educativa. En esta línea, Bolívar destaca que el liderazgo educativo es un factor de primer orden en la mejora de los resultados escolares.

Anteriormente, las maestras y los maestros que ocupaban un cargo de dirección en el centro escolar estaban principalmente asociadas a una mayor satisfacción del profesorado y con niveles más altos de aprendizaje del alumnado. Actualmente se concentran en los roles y en las estructuras que apoyan las prácticas educativas que deseamos (Sykes \& Elmore, 1989, p. 78). El problema pues se plantea al revés, tratar de ver qué prácticas de liderazgo son más adecuadas en un contexto organizativo y prescribir una lista de atributos exhortando a llevarlos a cabo. De acuerdo con Bolívar (2001), se pretende configurar los centros educativos como "sistemas más abiertos".

Bolívar (2010) defiende la tesis de que la mejora de la educación, entre otros factores, pasa por cambiar el modelo de dirección de las instituciones educativas. El modelo administrativo-burocrático de la dirección escolar, presenta graves déficits para incidir en la mejora de los resultados.

El líder, al tiempo que sus roles y responsabilidades se ven ampliados, tiene que actuar como un agente moral y social. En una cultura profesional de colaboración, es obvio que deben desarrollarse nuevas formas de liderazgo del profesorado más allá de la exclusiva preocupación del aula/grupo.

Lo nuevo aquí es la voluntad decidida de romper con la uniformidad predominante en los sistemas públicos y privados de la educación; por lo que se impele a que los centros escolares reflejen una identidad y personalidad propias.

Hasta no hace mucho tiempo, la cuestión del liderazgo estaba orientada a la organización y la gestión educativa (Lorenzo, 2005). A menudo, los 
planteamientos sobre este asunto provenían de otros contextos, en especial de la política y de la administración de empresas, ignorando la especificidad propia de la educación. Con ello se hacía un trasvase de las formas más eficaces de gestión basadas en una racionalidad técnica que, por sí misma, no han sido suficientes para garantizar una educación de calidad. El reto es apostar por un liderazgo personal capaz de aunar el bien común del grupo o institución educativa; un liderazgo personal que apueste por unos valores comunes educativos en sí mismos como guía para la acción que se desarrolla en un proceso dialógico y deliberativo, en el que todos los miembros participen en igual grado de condiciones para lograr un consenso común de valores e intereses (Mínguez Vallejos, 2013).

El liderazgo tiene relación con la conducción hacia el futuro. Castiñeira \& Lozano (2012) asocian el liderazgo a entornos de adaptación y procesos de cambio social en dichos entornos, especialmente, cuando existe por medio de las organizaciones el peligro de caer en el sistema establecido. En este sentido, para Mínguez Vallejos (2013), cualquier acción educativa comienza con la posibilidad de una transformación, teniendo en cuenta que el líder ético debe conducir al cambio, el cual debe traducirse en una mejora social (Giddens, 1989; Work, 1996).

Por su parte, Rojas \& Gaspar (2006) acotan que lo que importa en el liderazgo educativo es el aspecto humano. Si la acción de liderar está relacionada con la conducción de personas, nunca se insistirá lo suficiente en que el liderazgo, particularmente el educativo, está necesitado de centrarse en dos tareas básicas: a quiénes se conduce y para qué.

Se debe advertir que en las escuelas, especialmente, la dirección no presupone necesariamente tener capacidades o habilidades para el liderazgo, de la misma manera que el liderazgo no debe suponer forzosamente acceso a la dirección. Uno de los elementos claves que diferencia al líder de la dirección es el poder. De la dirección emana siempre el poder; en cambio, el líder se tiene que ganar la "autoridad". La investigación realizada por Díez, Terrón, Centeno \& Valle (2003), pone de manifiesto que esta confusión entre los dos términos es debida a que ambos están vinculados al ejercicio del poder en las organizaciones educativas. Cuando un centro actúa sólo en función de los resultados, es semejante a una organización empresarial que desarrolla un ejercicio de poder jerárquico e individualista. En este caso, cabe hablar de gestión o dirección, no de liderazgo. En este sentido, se 


\section{Mayoral et al. - Liderazgo en la Escuela Rural}

desarrolla un modelo masculino de dirección y así lo justifica muy acertadamente Santos Guerra (2000) cuando vincula dirección con orden y autoridad. Por otra parte, en contraposición al modelo tradicional, emergen formas de liderazgo más blandos. De acuerdo con Nye (2008), esta concepción invierte los términos del liderazgo tradicional, de tal manera que el estilo de liderazgo firme, competitivo y autoritario deja paso a un liderazgo integrador, dispuesto a aceptar y adaptar la conducta de los seguidores; de esta manera han emergido nuevos modelos de liderazgo tales como el distributivo, cooperativo, adaptativo, entre otros, más próximos a los modelos de liderazgo femenino (Chinchilla, 2007; Nye, 2008).

Centrándonos en Cataluña, la Fundación Jaume Bofill conjuntamente con el proyecto Entornos Innovadores de Aprendizaje de la OCDE, han desarrollado un proyecto que propone situar el aprendizaje en el centro de la cuestión educativa e insta a hacer un cambio de paradigma educativo que vincula dos aspectos: el aprendizaje y el liderazgo educativo (Martínez, Badia \& Jolonchi, 2013). De acuerdo con este proyecto, el liderazgo para el aprendizaje y los entornos innovadores de aprendizaje son conceptos dinámicos enfocados hacia el éxito escolar; el liderazgo para el aprendizaje es una modalidad de gestión y dinamización de los centros educativos que crea las condiciones para un posible buen desarrollo de la personalidad y del rendimiento académico del alumnado. Este estudio concluye que las organizaciones educativas que finalmente tienen éxito son aquellas que invierten en el crecimiento profesional de su personal (Martínez, Badia \& Jolonchi, 2013), y desarrollan un modelo de liderazgo orientado a las necesidades específicas del alumnado; es decir, de esta manera estaríamos hablando de un liderazgo para el aprendizaje.

\section{Metodología}

Durante mucho tiempo la escuela rural fue invisible a los ojos de la sociedad por no haber alcanzado los requisitos mínimos que requería la institución escolar; a partir de los años 60' se inicia un proceso de renovación pedagógica enfocado especialmente a la transformación de la escuela rural, que alcanza su zenit a principios de los 90', momento en que se inicia la transformación del sistema educativo y se constituye un nuevo modelo escolar con la aparición de las ZER (Zonas Escolares Rurales). Este modelo 
supone una nueva organización donde se distribuyen recursos humanos y materiales compartiendo un proyecto educativo común pero salvaguardando las singularidades de las escuelas (Ver: Samper, Burrial \& Sala, 2016). En el curso 2012-2013 había 88 escuelas ZER en las comarcas de Cataluña; los maestros y maestras que hemos seleccionado han participado de este proceso de renovación.

Con el fin de poder analizar la emergencia del liderazgo en los maestros y maestras de escuela rural, cómo se desarrolla y si existen unas características diferenciales entre los maestros y maestras líderes, se ha optado por la metodología cualitativa, más concretamente, el análisis de casos.

Walker (1983) define el estudio de casos como "el examen de un ejemplo en acción". Según este autor, el estudio de unos incidentes y hechos específicos y la recogida selectiva de información de carácter biográfico, de personalidad, de intervenciones y valores; permite al que lo realiza captar y reflejar los elementos de una situación que le dan significado. (Walker, 1983, p. 45)

De acuerdo con Vázquez \& Angulo (2003), el investigador mismo puede construir el caso, que emerge de la propia investigación. $O$ también a través del conocimiento pre-existente de una situación particular. El estudio de caso sigue la vía de la comprensión, de algo que está más allá para iluminar un problema o las condiciones que afectan al caso seleccionado. Nuestro objeto de estudio es precisamente comprender cómo se produce la transformación de los docentes en líderes.

El caso es importante porque es único. El investigador, en este estudio tiene que comprender lo que tiene de único y de particular. En esta investigación se ha encontrado que cada líder es único, cada uno tiene una potente base educativa, cada uno desarrolla proyectos diferenciados. Así, de acuerdo con Stake (1995), los docentes elegidos para nuestro estudio se corresponden a los criterios que ofrecen las mejores oportunidades de brindar conocimiento; cuestión que nos llevó a definirlos como líderes.

Es importante recordar que la selección de los casos no debe responder a casos típicos o representativos, ya que como bien indica Stake (1994) "no es probable que la muestra de un solo caso o unos casos sea una buena representación de otros". Por ello, este mismo autor señala que el primer criterio para la selección debe ser la máxima rendibilidad de aquello que aprendemos. Es decir, elegir los casos que nos den más oportunidad de 


\section{Mayoral et al. - Liderazgo en la Escuela Rural}

aprendizaje y entender con mayor profundidad los aspectos de nuestra investigación.

Inicialmente se realizó un primer contacto en 2012 con docentes de diferentes centros educativos rurales de Cataluña, lo cual permitió la identificación de dos maestros y dos maestras que ejercían de directores y directoras de sus escuelas y presentaban rasgos singulares que nos hicieron pensar a priori que reunían ampliamente las condiciones para ejercer con éxito el liderazgo. Tuvimos en cuenta nuestra experiencia y conocimiento de las escuelas rurales de Cataluña, así como el asesoramiento del Secretariado de Escuela Rural.

Los criterios de selección son: a) tener un amplio bagaje como docentes de escuela rural; b) tener experiencia como director/directora; c) haber implementado con éxito un proyecto de escuela singular y diferenciado y vinculado a su entorno; d) establecer relaciones estrechas y abiertas con la comunidad; e) ejercer el papel de líder reconocido por la comunidad educativa. Se observa que estos maestros y maestras desarrollan un elevado compromiso con la necesidad de transformar el sistema educativo.

El trabajo de campo se desarrolló durante seis meses del 2013 en las escuelas donde ejercían los maestros y maestras seleccionados, estas escuelas se encuentran situadas en las comarcas catalanas del Vallès Occidental, Bergadà, Osona y Alto Ampurdán.

Esta técnica nos permitió conocer la trayectoria personal y profesional de estas personas, así como sus percepciones y cómo se han construido como líderes y cómo ejercen su papel. Para ello, observamos diversas situaciones de la vida diaria del centro, y realizamos entrevistas en profundidad a los directivos y directivas. Entre otras cuestiones, preguntamos por la construcción del proyecto de escuela, los retos que han tenido que afrontar, sus concepciones sobre los modelos docentes y educativos, etc. Para el análisis de los datos obtuvimos unas categorías a partir de unas precategorías que habíamos elaborado previamente, teniendo en cuenta la fuente de información (observaciones o entrevistas).

En el desarrollo de esta investigación se siguieron los criterios nombrados por Stake (1995), como son la ética y la confidencialidad como aspectos relevantes. Para salvaguardar el anonimato de las personas y centros que han participado en esta investigación, les asignamos unos acrónimos, formados por dos letras mayúsculas, que construimos de la 
siguiente manera: la primera letra define el género ( $\mathrm{M}$ si es mujer, y $\mathrm{H}$ si es hombre), y la segunda letra (de las cuatro primeras letras del abecedario) designa el lugar de trabajo (escuela A, escuela B, escuela C, escuela D) y también el pueblo donde está ubicada la escuela (pueblo A, pueblo B, pueblo C, pueblo D).

\section{Maestros y Maestras Hacia el liderazgo}

A pesar de ser un tema crucial, el liderazgo educativo no está suficientemente explorado de manera empírica. Cuando se habla de liderazgo educativo se puede aludir a diferentes aspectos o facetas del espacio escolar. La investigación que se ha realizado se refiere a la transformación de estos cuatro maestros y maestras directivos y directivas en líderes, y se centra en tres aspectos: la constitución (formación) del liderazgo, el desarrollo de los proyectos educativos, y la diferenciación, si existe, entre liderazgos masculinos y liderazgos femeninos.

La cuestión esencial es cuál es el proceso que les ha conducido al liderazgo. En todos ellos y ellas existen motivaciones anteriores que les impelen a iniciar un período de búsqueda, los maestros y maestras seleccionados tienen en común la necesidad de transformar el sistema educativo tal y como ellos y ellas lo han vivido.

"Todo tiene una chispa que hace nacer un proyecto. Yo provenía de una realidad totalmente diferente... una escuela de ciudad... Yo hago el cambio... hago un proyecto nuevo". (HA)

"Yo decidí que para hacerlo de aquella manera no quería... no servía... y me fui a estudiar psicología a Barcelona... y me fui a trabajar a una escuela de educación especial... la libertad que me daba el hecho de no tener que trabajar con programas, con maneras concretas de hacer... yo creo que fue el primer paso a pensar que la escuela no tenía que ser lo mismo que yo había vivido en mi infancia... ni en aquel año en ..., que la escuela podía ser otra cosa...”. (MB)

"Como había crecido en una escuela de pueblo yo quería ser maestro en una escuela de pueblo...pero cuando llegué no tenía la mínima idea... tenía mis recuerdos como alumno pero no tenía ningún conocimiento de qué me encontraría, ni de qué tenía que hacer...". (HD) 


\title{
58 Mayoral et al. - Liderazgo en la Escuela Rural
}

El liderazgo no se improvisa. Construir un proyecto de escuela singular requiere un tiempo importante de reflexión, preparación, búsqueda y asesoramiento. Construir un nuevo proyecto requiere un grado de responsabilidad y compromiso importantes. Estos maestros y maestras a la vez que trabajan para transformar el medio educativo, transforman su visión de la educación y sus expectativas de futuro y las de la comunidad.

MB imagina un proyecto transformador donde los niños y las niñas pueden dar rienda suelta a su curiosidad y desarrollarla según sus intereses.

\begin{abstract}
"Cuando estaba en $\mathrm{X}$ (nombre de la escuela) una inspectora me vio $\mathrm{y}$ me dijo: ¿Por qué no te vas a B (nombre del pueblo donde está la nueva escuela) que allí estarás muy bien? Y le hice caso. Allí me encontré a una compañera, teníamos la misma visión de la escuela, de la infancia... de cuál tenía que ser nuestro papel [...] Antes habíamos intentado hacer una escuela un grupo de personas y había estado en contacto con Reggio Emilia. Escribí a Tonnuci en un determinado momento y me dijeron: "tendrías que ir a ver a Malagucci", lo fui a ver y me lo sacó de la cabeza, me encerró en un despacho y me dijo: "eso que tú estás intentado hacer... tiene que ser en la pública, porque es para todos los niños, no solamente para unos pocos". Y le hice caso. Volvimos aquí y dejamos la idea de la escuela y me fui hacia la pública... [...] Encontré dos compañeras y tuvimos que hablar muy poco, teníamos la misma idea y fuimos las tres por segunda vez a Región Emilia y tuvimos contacto con los parvularios, con la gente de allí, y volvimos más entusiasmadas de lo que estábamos, queríamos llevar a cabo un proyecto que nos ilusionaba pero que no tiene que ver nada con el actual". (MB)
\end{abstract}

Esta maestra percibe que las formas y maneras tradicionales que ella había vivido en las escuelas pueden transformarse en un nuevo concepto de escuela y de educación, y así paulatinamente crea un modelo nuevo e innovador.

Por otra parte, HD cuando llega al pueblo siente la necesidad de cambiar el modelo de escuela rural tradicional que encuentra, e inicia su proceso particular de búsqueda y transformación. 


\begin{abstract}
"Pensé... ¡caramba! Si esta gente es del Secretariado, son expertos y saben cómo funciona, tienes que ir y asistir a estas jornadas... y desde aquel momento me dije esta gente sabe cómo va la cosa... has de formar parte y así fue como comencé... y busqué también información...así fue como descubrí Freinet... [...] Lo que me impactó más fueron las diferentes técnicas de Freinet. Y aquí pensé, esto es parte de la solución de lo que estoy buscando, que tenía que ver con los planes escolares o la imprenta escolar, que hace que todo el trabajo de la escuela sea significativo [...]. Fui buscando más información. [...]A partir de aquí fuimos construyendo el proyecto de la ZER (...) A mí me sorprendió que se trabajara con libros y a partir de esto fuimos experimentando con todo tipo de metodologías". (HD)
\end{abstract}

Para MC el proceso es similar. Ella ha adquirido experiencias diversas a través de su participación en los Movimientos de Renovación Pedagógica, su activismo político y su experiencia docente, habilidades que le sirven para construir un nuevo proyecto de escuela.

"Yo llego a C (nombre del pueblo) con un bagaje de haber trabajado en dos escuelas y un bagaje personal de haber estado vinculada $\mathrm{y}$ todavía estoy, en los movimientos de renovación pedagógica... el trabajo en equipo, la colaboración con la gente son dos aspectos fundamentales que me llevo conmigo cuando llego a B. (nombre del pueblo) y me encuentro una escuela que he de montar prácticamente yo sola y con compañeros que un principio por dificultades personales no colaboran, después sí”. (MC).

HA trabajaba en la escuela privada como maestro de educación infantil, pero el trabajo rutinario de la escuela no le satisfacía y se preguntaba: ¿Toda la vida tendré que explicar lo mismo? Decide iniciar nuevos estudios, y asistir a los cursos de formación del ICE de la Universidad Autónoma de Barcelona. Cambia de la escuela privada a la escuela pública. Después, el Departament d'Ensenyament de la Generalitat de Catalunya le encarga la realización de un nuevo proyecto para recuperar la escuela A (nombre de la escuela), y acepta el reto.

"Yo provenía de una realidad totalmente diferente, una escuela de ciudad, de 4 líneas, hago el cambio [...] Tengo la necesidad de 


\section{Mayoral et al. - Liderazgo en la Escuela Rural}

compartir mi inquietud con compañeros del grupo ICE [...] En este grupo encuentro conexiones, encuentro gente que había trabajado las ideas que yo tenía pero muy elaboradas [...] Justo cuando hacía 6 meses que estaba, se me propone el reto de abrir la escuela A (...) Ante este reto yo me documento, leo y escribo las líneas de lo que tendría que ser el proyecto de escuela, y lo nombraba la Autopista de la escuela; un proyecto que tuviera una dirección muy bien definida pero que fuera suficientemente amplia para dar cabida a diferentes miradas. El reto empezó por buscar las personas que quisieran formar parte de este proyecto, formar alumnos críticos que tuvieran en cuenta el entorno que nos envuelve, son huellas que tuvimos en cuenta en este primer momento. No fue un camino fácil. Yo diría que hay una esencia que no tiene que cambiar pero hay una mirada hacía aquello que aprendemos y cómo aprendemos, una reflexión”. (HA)

HA desarrolla su proyecto en una zona rural con dos comunidades bien diferenciadas: los habitantes de toda la vida y los recién llegados. Su implicación con la comunidad es elevada y tiene claro desde un principio que la primera acción que debe acometer es trabajar para cohesionar las dos poblaciones. HA deberá ganarse la confianza del pueblo y posteriormente la confianza de los padres. El primer paso es participar de la vida cotidiana del pueblo, sus fiestas, su actividad cultural y mostrar pro-actividad.

Otro reto es ganarse a las familias, para ello las invita a la escuela y las pone en situación de aprendizaje realizando actividades similares a las que hacen sus hijos e hijas. HA analiza también los niveles de instrucción de la población, que son de nivel medio-bajo, dado que se plantea que si los padres no han tenido éxito escolar van a tener dificultades para entender su proyecto; es decir, se pregunta cómo puede implantar unas nuevas metodologías si las familias no comprenden qué se hace en la escuela. Para ello los espacios de la escuela se abren a la comunidad; si los padres y las madres se apropian de los espacios escolares, todo va a revertir en una mejora y comprensión de estos espacios y del trabajo escolar. Otro requisito importante fue buscar un equipo que se quisiera adherir al compromiso. Algunos de los maestros y maestras líderes han tenido la oportunidad de participar en el diseño de la escuela, creando unos espacios adecuados para desarrollar el proyecto. Otros, en cambio, no han gozado de esta oportunidad, pero han sido capaces de ordenar los espacios según las necesidades de la institución. Ya se trate de escuelas en viejos edificios de 
antiguas naves industriales o escuelas de nueva construcción, todas ellas tienen en común la movilidad del alumnado, los espacios versátiles, una relación estrecha con el exterior, etc. Por ejemplo, en la escuela de B se tiene un espacio a la entrada de la escuela en forma de anfiteatro de usos múltiples para el encuentro, la asamblea de alumnado, de familias, para observar y trabajar en proyectos de innovación, gestionar demandas y conflictos. El anfiteatro es un punto diario de encuentro que invita a hablar, pensar o imaginar.

\begin{abstract}
"Este local es fruto de nuestra intervención como maestros... entonces pedimos al arquitecto que nos hizo caso, que necesitábamos un espacio colectivo que nos ha de servir para hacer presentaciones, exposiciones, que tiene que ser un lugar donde podamos hacer obras de teatro, también será el gimnasio, etc. Después unas aulas... que puedes hacer el aula más cerrada, más abierta en función de qué necesidades y puedes repartir el alumnado según qué actividad estés haciendo". (MC)

"El edificio es desastroso pero esto le da también por otro lado la calidez de un edificio viejo que no está pensado para unas condiciones concretas, unas determinadas finalidades, pero esto también ha permitido un espacio familiar, que el cambio no fuera solamente de unas pedagogías concretas sino que el edificio acompañara también en este tipo de trabajo (...) tenemos en el proyecto de escuela que los espacios acompañan a las personas". (MB)
\end{abstract}

\title{
Proyectos: Estrategias, Motivaciones y Valores
}

Maestros y maestras desarrollan un modelo de liderazgo con visión de futuro, en el sentido de ser imaginativos e intuitivos para transformar la realidad, del mismo modo apoyan su trabajo en la reconstrucción de la comunidad escolar a partir del diálogo y la confianza con la comunidad; las familias independientemente de sus orígenes sociales y étnicos se sienten acogidas, seguras e integradas. Estas escuelas mantienen una colaboración estrecha y comprometida con su medio social y natural. Por ejemplo, para $\mathrm{MC}$ el trabajo escolar tiene sentido cuando se devuelve a la sociedad.

"Con esta línea yo pienso que hemos ido trabajando una relación muy directa con el pueblo, algunas veces a través de proyectos muy 


\section{Mayoral et al. - Liderazgo en la Escuela Rural}

ambiciosos como el pueblo educador donde la escuela estaba abierta a la comunidad e intentaba ser un espacio de encuentro de la comunidad". (MC)

"Llegó un punto al $2006 \ldots$ porque la escuela había ido creciendo sobre todo debido a la atención a la diversidad, hace que la escuela se conozca y las familias se planteen llevar a sus hijos, rebotados de escuelas más convencionales, a una escuela un poco diferente, la escuela se fue llenando... fue creciendo...". (MB)

Tanto los hombres como mujeres desarrollan proyectos propios. Las escuelas, además, son espacios de investigación y crecimiento personal. Tienen en común que gozan de un buen número de seguidores que va más allá de la comunidad escolar.

"Hemos generado una escuela con ADN que en estos 7 años nos ha hecho crecer... si no lo hiciéramos, seriamos una escuela que tendría un proyecto, pero no seriamos una escuela que avanza, que aprende". (HA)

"Yo pasé a ser directora y como tengo una especie de pasión por las ciencias quizás se contagió esta especie de visión al resto del equipo, a los maestros, a los niños, una visión más científica”. (MB)

"La escuela no somos solamente los maestros, están los padres, están los abuelos, están los vecinos, que tienen mucha información que nos pueden aportar en nuestro día a día”. (MC)

El liderazgo implica diferenciación. A pesar de que estas y estos líderes comparten el compromiso para transformar la escuela y unos valores sobre cómo debe ser la educación, el desarrollo de cada centro es diferente, la personalidad de cada uno hace que estos proyectos de escuela sean singulares.

HA construye un proyecto educativo que se basa en cuatro pilares: una escuela de la comunicación, una escuela del bienestar (se disfruta de lo que se aprende), una escuela que aprende (aprende el alumnado, el profesorado y la comunidad), una escuela abierta a la comunidad. Su metodología se basa en la creación de redes de significado a partir del método científico.

Por el contrario, MB basa su proyecto en una nueva forma de aprender las matemáticas, partiendo de que el conocimiento matemático está en todas partes. Las matemáticas son el eje central a partir del cual se conecta el 
conjunto de conocimientos. El método de trabajo consiste en crear grupos de investigación sobre diferentes temáticas, la base es el método científico con el que se debe razonar, ordenar, clasificar, jerarquizar, plantearse nuevas preguntas y no dar por cerradas las respuestas.

Para MC, los valores que desarrolla su proyecto se articulan a partir de tres conceptos: la sostenibilidad, el medio ambiente y la democracia; todo bajo un potente paraguas de carácter social. El nivel de implicación con la comunidad es muy elevado. Se basa en el método científico, en su trabajo cotidiano busca el equilibrio entre la teoría y la práctica, desarrolla un profundo respeto por la comunidad. Un aspecto importante es reconocer el entorno, situar la práctica y vincularla con el conocimiento escolar.

"Lo primero que hago es reconocer el entorno, buscar qué tiene y de qué me sirve a mis intereses y a los de la población, el medio físico y social de las personas, el trabajo que han desarrollado... los proyectos tienen una parte práctica que los alumnos puedan tocar aquello que hacen $y$ ver de primera mano que la teoría viene de una experimentación, de un análisis de los elementos". (MC)

Para HD, un aspecto clave es la construcción de relaciones abiertas con el entorno. La escuela es además un importante eje de dinamización de la comunidad y del pueblo. HD ha logrado recomponer la escuela a partir de un trabajo atractivo, capacidad de empatía y un potente diálogo con todos $\mathrm{y}$ cada uno de sus habitantes. Ha logrado recomponer la escuela y ganarse la confianza de sus habitantes.

"Yo pienso que uno de los ejes fundamentales es cómo la escuela integra este medio y cómo el medio integra la escuela...". (HD)

"Desde los inicios queríamos una escuela que aprendiera, no solamente unos niños que aprendieran, unos maestros que aprendieran, sino una escuela que aprendiera y cuando explicábamos la escuela a las familias en el 2007, cuando se tenían que matricular, una frase que les hacíamos llegar era: "queremos una escuela en la cual los niños no hagan lo que quieran, sino que estimen aquello que hacen". Hacer crecer el deseo por aprender. Los rasgos que no queremos perder de vista es el proyecto educativo". (HA) 
"Una fuerte vinculación con el entorno próximo, la vida está fuera, los conocimientos están fuera, dentro podemos crear los contactos...”. (MB)

"Reflexionando dentro del equipo nos hemos dado cuenta que la investigación es la única manera de aprender, por tanto en la escuela, sí, la investigación se puede hacer a partir del método científico dentro del trabajo escolar...". (MB)

\section{Género y Liderazgo: Procesos de Desdiferenciación}

Las relaciones que mantienen los y las líderes se alejan del paradigma convencional, por una parte, los maestros líderes no responden al modelo tradicional de rigidez, autoridad e incentivos. Así, son dialogantes, desarrollan un modelo empático que genera confianza y buen hacer. Por otro lado, las mujeres líderes se alejan de los tópicos y estereotipos, dialogan, saben tomar decisiones y no rehúyen de los conflictos.

Para acercarnos más al desarrollo y conocimiento de estos líderes tomamos como ejemplo el Claustro pedagógico, una de las actividades formales que se desarrollan semanalmente en la escuela A y que tiene la función de poner en común todo lo que acontece académicamente en el centro. Tarea que implica: diálogo, reflexión, coordinación, distribución de tareas, orientación y reorientación. Se trata de un ejercicio de liderazgo compartido, distributivo y colaborativo, ya que requiere la implicación de todo el equipo docente; es decir, si el Claustro pedagógico no funciona, la escuela tampoco funciona. Este modelo se acerca al modelo blando que propone Nye (2008).

Asimismo, HD ha logrado con esfuerzo y habilidad, generar elevadas dosis de confianza por parte de la comunidad en la escuela. Ha logrado recomponer los vínculos escuela-comunidad de tal manera que la escuela deviene un núcleo de referencia del pueblo. A título de ejemplo, en dependencias de la escuela se han creado espacios compartidos como la biblioteca. Allí se ha formado un grupo de lectura de adultos, que a menudo recibe visitas de alumnos de todas las edades para leer conjuntamente.

Las maestras líderes se acercan también a estos modelos blandos y dialogantes. Dos principios están en la base de su trabajo: la construcción de sentido de lo que se hace y para qué se hace y una fuerte convicción de justicia social. MB insiste que "el profesorado tiene que dar al máximo y el 
alumnado debe de llegar al máximo de su capacidad"; recuerda MB que "en la escuela no hay techo". Para MC la implicación social con la que plantea sus actividades cotidianas y su relación con el medio físico y social, constituyen dos pilares fundamentales de su proyecto. La pro-actividad que muestra MC tanto en el medio escolar como en la comunidad constituye un acicate del saber ser y hacer de una maestra comprometida con su trabajo.

\section{Conclusiones}

Tal como sugiere la metodología de análisis de casos, las escuelas analizadas son únicas en su contexto. A pesar de que los maestros y las maestras líderes comparten unos principios educativos similares sobre lo que debe ser la educación, su desarrollo es notablemente distinto. Esta diferencia viene determinada por las habilidades que poseen cada uno de estos líderes.

Se ha podido observar cómo en un momento dado de su trayectoria laboral, estos y estas líderes ponen de manifiesto su necesidad de transformación del sistema educativo. Inician un periodo de estudio, viajes, conversaciones sobre el tema. Las vías de construcción de los proyectos son notablemente diferentes para cada uno de ellos y ellas.

Los principios sobre los que construyen sus escuelas son comunes: partir de los intereses de los niños y las niñas, así como del método científico como base del trabajo, una especial atención en los proyectos de investigación y en el trabajo en equipo.

Son escuelas con una elevada movilidad, con espacios versátiles, donde se fomenta la motivación por el conocimiento y el trabajo bien hecho. En contrapartida a estos principios, sus proyectos son radicalmente diferentes: van desde el construccionismo y el trabajo de redes, la matemática como eje fundamental del trabajo escolar, la ecología y los medios físico y social, hasta la recuperación de la escuela como elemento fundamental para el desarrollo de la vida rural.

Son proyectos muy creativos que requieren de un elevado grado de dedicación, lo que implica que no sólo el alumnado aprende sino también los docentes; es decir, la dedicación de los maestros y maestras va más allá de los aspectos de coordinación, y se ven obligados a diseñar nuevos elementos que puedan contribuir a la orientación y desarrollo de los proyectos del alumnado. 


\section{Mayoral et al. - Liderazgo en la Escuela Rural}

Las programaciones curriculares homogéneas no sirven al alumno que está desarrollando un proyecto de investigación, por el contrario, los y las docentes deben dejar registrado, a menudo con posterioridad, todo el desarrollo y progresos del alumnado.

En este sentido, si la Administración facilitara la composición de equipos de trabajo con una preparación básica y buena disposición, mejoraría el trabajo escolar. Otra dificultad es la falta de motivación de los maestros y maestras por trabajar en una escuela rural. A veces, el desplazamiento a zonas rurales supone un inconveniente añadido.

Finalmente, retomamos la eterna discusión sobre las diferencias de liderazgo según el género. Cabe destacar que en los años que se ha realizado este proyecto no se han constatado diferencias de género. Los y las líderes estudiados se caracterizan por su capacidad de comunicación. Tanto en hombres como en mujeres, no se observan rasgos autoritarios, más bien se dialoga, se reflexiona, se plantean alternativas. Ambos tienen un marcado carácter social y una gran capacidad de ejecución.

De acuerdo con Nye (2011), a medida que las sociedades alcanzan mayor calidad democrática, tienden también a la democratización del poder; es decir, se aproximan a lo que este autor llama poder blando. El poder blando es más persuasivo, controla mejor las emociones y tiende a reconocer y a escuchar a sus seguidores. Se observa en este estudio sobre el liderazgo en las escuelas rurales, como se aproximan los estilos o modelos entre hombres y mujeres, no sólo en la comunicación sino también en el desarrollo de las actividades cotidianas en las aulas.

Finalmente se observa una proliferación y diferenciación de categorías relacionadas con el liderazgo que dificultan el reconocimiento de los verdaderos líderes, aunque cabe reconocer que algunos directivos y directivas desarrollan tareas excelentes. En el caso que nos ocupa, los y las líderes que hemos analizado se distinguen por: a) la solidez y fundamentación de sus conocimientos que les permiten imaginar nuevas formas educativas dotándolas de sentido. Una observación: todos y todas llegan al liderazgo a edades adultas; b) el grado elevado de compromiso personal con la necesidad de transformar el sistema educativo; c) la realización de un proyecto global que abarca todos los ámbitos escolares y que se encuentra vinculado a procesos de cambio e innovación. Todos los actos son fruto del diálogo previo y permanente entre docentes, discentes y 
familias. En este sentido la implicación del medio es muy importante, el "aprender a aprender" adquiere nuevo significado; d) la inspiración, el conocimiento y el uso del método científico contribuyen a la creación de proyectos escolares interesantes; estas escuelas recogen el espíritu del proyecto humanista y de la racionalidad.

\section{Referencias}

Asensio Aguilera, J.M. (2013). El docente y la idea de Liderazgo. En J. Argos \& P. Ezquerra (Eds.), Liderazgo y Educación. Santander: Ediciones Universidad de Cantabria.

Bolívar, A. (2001). Liderazgo educativo y reestructuación escolar.

Conferencia en el I Congreso Nacional sobre Liderazgo en el Sistema Educativo Español. (Córdoba, 28-31 de marzo de 2001). Actas del Congreso, pp. 95-130. Córdoba: Departamento de Educación de la Universidad de Córdoba.

Bolívar, A. (2010). El liderazgo educativo y su papel en la mejora: Una revisión actual de sus posibilidades y limitaciones. Psicoperspectivas, 9(2), 9-33.

Bolívar, A. (2012). Políticas actuales de mejora y liderazgo educativo. Archidona (Málaga): Ed. Aljibe.

Castiñeira, A. \& Lozano, J.M. (2012). El Poliedro del liderazgo. Una aproximación a la problemática de los valores en el liderazgo. Barcelona: ESADE.

Chinchilla, N. (2007). Dueños de nuestro destino. Como conciliar la vida profesional, familiar y personal. Barcelona: Ariel.

Díez, E.J.; Terrón, E.; Centeno E.; \& Valle, B. (2003). El liderazgo femenino y su ejercicio en las organizaciones educativas. Revista Iberoamericana, 2003, 31.

Frederickson, H.G. \& Smith, K.B. (2003). The public administration theory primer. En J. Argos \& P. Ezquerra (Eds.) (2013), Liderazgo y Educación. Santander: Ediciones Universidad de Cantabria.

Fry, B.R. (1989). Mastering public administration. From Max Webwe to Dwight Waldo. En J. Argos J., \& P. Ezquerra (2013), Liderazgo y Educación. Santander: Ediciones Universidad de Cantabria. 
Gallego, R.; González, S.; \& Brugué, Q. (2011). Entre la dirección y el liderazgo en los centros escolares: Diseño de una investigación. Ponencia al X Congreso Asociación Española de Ciencia Política y de la Administración (AECPA). Recuperado de http://www.aecpa.es/uploads/files/modules/congress/10/papers/110.pd $f$

Giddens, A. (1989). Sociology. Cambridge: PolityPress.

Guillem M. (1994). Models of management. Work, authority and organitazion in a comparative perspective. En J. Argos \& P. Ezquerra (eds.) (2013), Liderazgo y Educación. Santander: Ediciones Universidad de Cantabria.

Lorenzo, M. (2005). El liderazgo en las organizaciones educativas. Revisión y perspectivas actuales. Revista Española de Pedagogía, 63, 232, $367-$ 388.

Losada, C. (2007). Liderar en l'àmbit públic. En F. Longo, \& T. Ysa (eds), Els escenaris de la gestió pública del segle XXI. Barcelona: Escola d'Administració Pública.

Martínez-Muñoz, M.; Badia-Pujol, J. \& Jolonchi-Anglada, A. (2013).

Lideratge per a l'aprenentatge. Estudis de cas a Catalunya.

Barcelona: Fundació Bofill.

Mínguez Vallejos (2013). Liderazgo, Ética y Educación. En J. Argos \& P. Ezquerra (eds.) (2013), Liderazgo y Educación. Santander: Ediciones Universidad de Cantabria.

Mintzberg, H. (1983). Rounding out the manager's job. Soloam

Management Review, 36 (1), 11, 25. En J. Argos \& P. Ezquerra (eds.) (2013), Liderazgo y Educación. Santander: Ediciones Universidad de Cantabria.

Nye, J. (2008). Las cualidades del lider. Editorial Paidós.

OCDE (2009). Innovating to Learn, Learning to Innovate. París. OCDE.

OCDE (2009). Creating Effective Teaching and Learning Environments:

First Results from TALIS. Paris: OCDE Publishing.

Rojas, A. \& Gaspar, F. (2006). Bases educativas del liderazgo. Publicado por la Oficina Regional de Educación de la UNESCO para América Latina y el Caribe. OREALC/UNESCO. Santiago. 
Samper, L., Burrial, X. \& Sala, T. (2016). Escoles, pobles i famílies. Noves perspectives sobre l'escola rural a Catalunya. Ediciones de la Universitat de Lleida.

Santos Guerra M. (2000). "Yo tengo que hacer la cena". La mujer y el gobierno de los centros escolares. En M.A. Santos Guerra (coord.), El harén pedagógico. Perspectivas de género en la organización escolar (pp. 53-69). Barcelona: Graó.

Stake, R.E. (1994). Case Study. En N. K. Denzin \& Y.S. Lincoln (eds.), Handbook of Qualitative Research (pp. 236-247). London: Sage.

Stake, R.E. (1995). The Art of Case Study. London: Sage.

Sykes, G. \& Elmore, R. E. (1989). Making schools manageable: policy and administration for tomorrow's schools. En J. Hannaway y R. Crowsom (eds.), The politics of reforming-school Administration (The 1988 Yearbook of the politics of Education Association) (pp. 77-94). Londres: Falmer Press.

Vázquez Recio, R. \& Angulo Rasco F. (2003). Introducción a los estudios de casos. Los primeros contactos con la investigación etnográfica. Málaga: Ediciones Aljibe.

Velasco, H. \& Díaz de Rada, A. (1997). La lógica de la investigación etnográfica. Un modelo de trabajo para etnógrafos de la escuela. Madrid: Trotta.

Walker, R. (1983). La realización de estudios de casos en educación. Ética, teoría y procedimientos. En W. B. Dockrell, \& D. Hamilton, (Comps.), Nuevas reflexiones sobre la investigación educativa (pp. 42-82) Madrid: Narcea.

Work, W. (1996). La dirección de una variada plantilla de personas. En F. Hesselbein; M. Goldsmith \& R. Beckhard (1996) El líder del futuro. The Drucker Foundation (capítulo 7). Bilbao: Ediciones Deusto S.A 
70 Mayoral et al. - Liderazgo en la Escuela Rural

Dolors Mayoral Arqué es profesora en la Universitat de Lleida

Joana Colom es profesora en la Universidad de las Islas Baleares

Olga Bernad es profesora en la Universitat de Lleida

Teresa Torres González es profesora en la Universitat de Lleida

Dirección de contacto: dmayoral@geosoc.udl.cat 